\title{
Thoracic osteomyelitis and epidural abscess formation due to cat scratch disease: case report
}

\author{
David Dornbos III, MD, ${ }^{1}$ Jocelyn Morin, MD, ${ }^{2}$ Joshua R. Watson, MD, ${ }^{3}$ and Jonathan Pindrik, MD ${ }^{1,4}$ \\ Departments of ${ }^{1}$ Neurological Surgery and ${ }^{2}$ Family Medicine, The Ohio State University Wexner Medical Center; ${ }^{3}$ Department \\ of Pediatrics, Nationwide Children's Hospital and The Ohio State University; and ${ }^{4}$ Division of Pediatric Neurological Surgery, \\ Nationwide Children's Hospital, Columbus, Ohio
}

\begin{abstract}
Osteomyelitis of the spine with associated spinal epidural abscess represents an uncommon entity in the pediatric population, requiring prompt evaluation and diagnosis to prevent neurological compromise. Cat scratch disease, caused by the pathogen Bartonella henselae, encompasses a wide spectrum of clinical presentations; however, an association with osteomyelitis and epidural abscess has been reported in only 4 other instances in the literature. The authors report a rare case of multifocal thoracic osteomyelitis with an epidural abscess in a patient with a biopsy-proven pathogen of cat scratch disease. A 5-year-old girl, who initially presented with vague constitutional symptoms, was diagnosed with cat scratch disease following biopsy of an inguinal lymph node. Despite appropriate antibiotics, she presented several weeks later with recurrent symptoms and back pain. Magnetic resonance imaging revealed 2 foci of osteomyelitis at T-8 and T-11 with an associated anterior epidural abscess from T-9 to T-12. Percutaneous image-guided vertebral biopsy revealed $B$. henselae by polymerase chain reaction analysis, and she was treated conservatively with doxycycline and rifampin with favorable clinical outcome.
\end{abstract}

http://thejns.org/doi/abs/10.3171/2016.7.PEDS1677

KEY WORDS cat scratch disease; osteomyelitis; epidural abscess; Bartonella henselae; infection

$B$ artonella henselae, the causative organism of cat scratch disease, typically presents with lymphadenopathy neighboring a cat scratch or bite. While the rise of specific antibodies may suggest infection by the causative organism, serology may lag in the initial stages. As a small, slow-growing, intracellular gram-negative bacillus, $B$. henselae typically requires polymerase chain reaction (PCR) evaluation of target genes for diagnosis, due to frequent nondiagnostic findings of direct Gram stain and culture. Cat scratch disease often follows an indolent course with spontaneous resolution. However, this organism may cause visceral infections or osteomyelitis on rare occasions. ${ }^{5}$ Few prior reports of cat scratch disease in association with spinal osteomyelitis and epidural abscess formation have been reported. ${ }^{1,6,7,11}$ With the paucity of published findings, treatment approaches still vary significantly between surgical and nonsurgical interventions. We report the third biopsy-proven case of $B$. henselae spinal epidural abscess formation and review treatment strategies for this rare clinical entity.

\section{Case Report}

History and Examination

A 5-year-old girl presented after 10 days of significant fevers $\left(40^{\circ} \mathrm{C}\right)$, emesis, inguinal lymphadenopathy, and diffuse abdominal pain. Epidemiological history revealed exposure to home pets including dogs, cats, and recently born kittens, but no reported occurrence of cat scratch or bite. Laboratory evaluation showed an initial peripheral smear white blood cell count of $11,500 / \mathrm{mm}^{3}$ with $50 \%$ neutrophils, $35 \%$ lymphocytes, $13 \%$ monocytes, and $1 \%$ eosinophils. Inflammatory markers, including erythrocyte sedimentation rate (ESR) and C-reactive protein (CRP) level,

ABBREVIATIONS CRP = C-reactive protein; $E S R=$ erythrocyte sedimentation rate; $\mathrm{PCR}=$ polymerase chain reaction.

SUBMITTED February 10, 2016. ACCEPTED July 6, 2016.

INCLUDE WHEN CITING Published online September 23, 2016; DOI: 10.3171/2016.7.PEDS1677. 

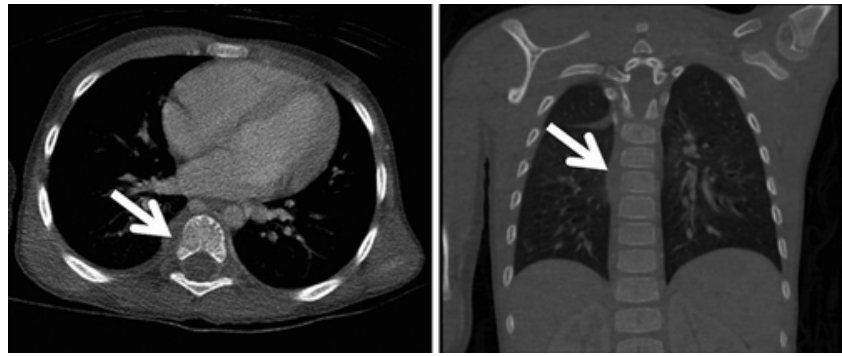

FIG. 1. Axial (left) and coronal (right) chest CT scans demonstrating a right paraspinal abscess (arrow) and cortical destruction of the adjacent T-8 vertebral body.

were elevated at $54 \mathrm{~mm} / \mathrm{hr}$ and $5.3 \mathrm{mg} / \mathrm{dl}$, respectively. Despite unremarkable blood and urine cultures, serum immunoglobulin assay revealed a profile consistent with cat scratch disease (immunoglobulin $\mathrm{G}>1: 128$, immunoglobulin $M>1: 32$ ). Treatment recommendations included outpatient antibiotic therapy with azithromycin. Four days following discharge, the patient was readmitted for persistent fevers, irritability, decreased appetite, and severe epigastric abdominal pain. Abdominal and pelvic CT revealed an inguinal abscess that demonstrated PCR evidence of $B$. henselae following incision and drainage. Despite initiation of a new course of azithromycin, the patient continued to experience persistent fevers and epigastric pain, with laboratory evidence of increasing inflammatory markers (ESR $58 \mathrm{~mm} / \mathrm{hr}$, CRP $23.7 \mathrm{mg} / \mathrm{dl}$ ).

\section{Imaging and Biopsy}

Chest CT revealed a lytic lesion of the T-8 vertebral body with loss of normal cortical bone and concern for an adjacent paraspinal abscess (Fig. 1). Spinal MRI subsequently revealed multifocal thoracic vertebral osteomyelitis at T-8 and T-11, prevertebral soft-tissue enhancement from the T-6 through L-1 levels, and an epidural abscess centered at the T-11 vertebral body (Fig. 2). Despite these radiological findings, the patient remained neurologically intact. A CT-guided biopsy of the T-8 vertebral body revealed $B$. henselae by PCR, confirming the diagnosis.

\section{Disease Course and Treatment}

Antibiotic therapy was switched from azithromycin to a combination intravenous regimen including doxycycline and rifampin for presumed vertebral osteomyelitis due to $B$. henselae. After 6 days of intravenous therapy and resolution of clinical symptomatology, doxycycline and rifampin were switched to oral administration, allowing outpatient therapy. Rifampin was continued for a total of 3.5 weeks, and doxycycline was continued until infectious markers (ESR and CRP) normalized following 6.5 weeks of therapy. MRI at the completion of antibiotic therapy revealed no evidence of the epidural abscess and nearcomplete resolution of the T-8 and T-11 osteomyelitis (Fig. 3 ), with a plan for further imaging 6-8 weeks following antibiotic completion.

\section{Discussion}

Cat scratch disease represents an uncommon cause of
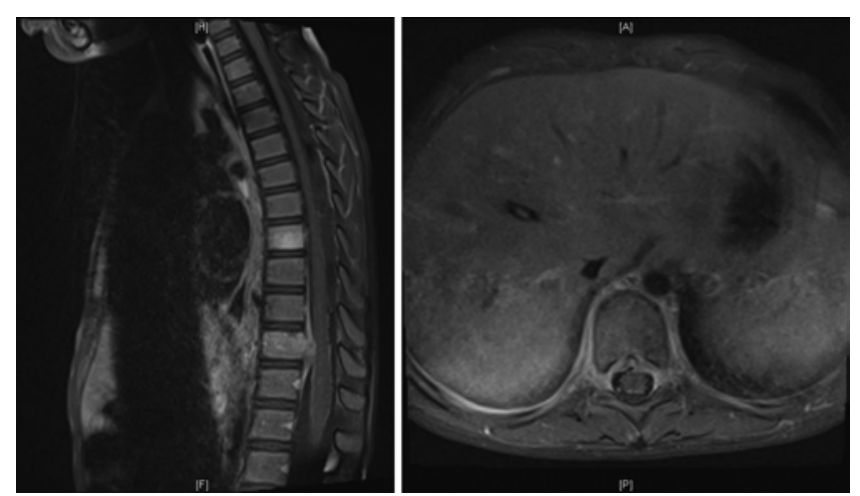

FIG. 2. Sagittal (left) and axial (right) MR images of the thoracic spine revealing abnormal contrast enhancement of the T-8 and T-11 vertebral bodies, consistent with osteomyelitis. Additionally, an epidural abscess posterior to the T9-12 vertebral bodies caused minimal thecal sac compression.

vertebral osteomyelitis, with limited attention in prior literature. The association of this pathogen with an epidural abscess has been described in only 4 prior cases ${ }^{1,67,11}$ only 2 of which were proven with a surgical biopsy or aspiration of the affected vertebral body or abscess (Table 1). ${ }^{7,11}$ Often, cat scratch disease may resolve spontaneously or with medical management; however, the association with a compressive epidural abscess may necessitate surgical intervention. Spinal epidural abscesses have historically been associated with high rates of morbidity and mortality, especially in the pediatric population due to delayed diagnosis and nonspecific symptoms at disease onset. ${ }^{6}$ However, diagnosis and management have improved significantly with enhanced imaging modalities and new antibiotic regimens. The presence of neurological deficits referable to an epidural abscess typically warrants surgical decompression followed by antibiotic treatment; however, in the absence of neurological findings, antibiotic treatment alone without open surgery represents adequate therapy in most cases. ${ }^{6}$

Abdel-Haq et al. reported the first case of an epidural abscess in association with cat scratch disease. ${ }^{1}$ A 5-yearold boy was found to have a midthoracic epidural abscess
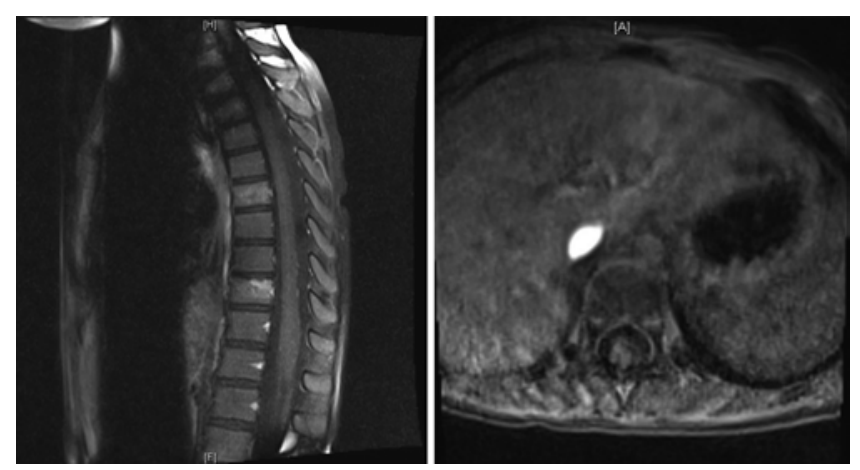

FIG. 3. Sagittal (left) and axial (right) posttreatment MR images of the thoracic spine demonstrating minimal enhancement of the T-8 and T-11 vertebral bodies and complete resolution of the previously identified epidural abscess. 
TABLE 1. Case reports in the available literature revealing a spinal epidural abscess secondary to cat scratch disease

\begin{tabular}{|c|c|c|c|c|c|}
\hline Authors \& Year & $\begin{array}{c}\text { Age (yrs), } \\
\text { Sex }\end{array}$ & Location & Pathogen Diagnosis & Empiric Treatment & Definitive Treatment \\
\hline Abdel-Haq et al., 2005 & $5, M$ & Thoracic & Serology & Vancomycin \& ceftriaxone* & $\begin{array}{l}\text { Clarithromycin (initial), trimethoprim/ } \\
\text { sulfamethoxazole (final) }\end{array}$ \\
\hline $\begin{array}{l}\text { Hussain \& Rathore, } \\
2007\end{array}$ & $3, M$ & Thoracic & $\begin{array}{l}\text { Serology, PCR (aspira- } \\
\text { tion) }\end{array}$ & Cefazolin* & $\begin{array}{l}\text { Clindamycin \& gentamicin (initial), tri- } \\
\text { methoprim/sulfamethoxazole (final) }\end{array}$ \\
\hline Tasher et al., 2009 & $5, M$ & Cervical & $\begin{array}{l}\text { Serology, PCR (sur- } \\
\text { gery) }\end{array}$ & $\begin{array}{l}\text { Cloxacillin, gentamicin, \& ceftriax- } \\
\text { one }^{*}\end{array}$ & $\begin{array}{l}\text { Gentamicin \& rifampin (initial), azithro- } \\
\text { mycin \& rifampin (final) }\end{array}$ \\
\hline Hawkins \& Bolton, 2013 & $10, F$ & Thoracic & Serology & Vancomycin, gentamicin, \& rifampin* & Gentamicin \& rifampin \\
\hline Current study & $5, \mathrm{~F}$ & Thoracic & Serology, PCR (biopsy) & Azithromycin† & Doxycycline \& rifampin \\
\hline
\end{tabular}

* Empiric treatment for vertebral osteomyelitis and/or epidural abscess prior to diagnosis of $B$. henselae.

$\dagger$ Treatment for $B$. henselae lymphadenitis prior to diagnosis of vertebral osteomyelitis and epidural abscess.

with adjacent vertebral osteomyelitis, requiring initial broad-spectrum antibiotic coverage with vancomycin and ceftriaxone and subsequent surgical decompression. While intraoperative cultures remained negative, serum immunoglobulin $\mathrm{G}$ titers suggested $B$. henselae infection. The ultimate treatment regimen consisted of clarithromycin for 2 weeks, followed by trimethoprim/sulfamethoxazole for a total 10-week antibiotic course.

Two prior case reports have identified $B$. henselae infection by PCR analysis of fluid obtained directly from the affected vertebrae. Hussain and Rathore described a 3 -year-old boy found to have thoracic osteomyelitis with an abscess in the adjacent psoas muscle. ${ }^{7}$ The initial antibiotic regimen consisted of intravenous clindamycin and gentamicin; however, follow-up scans revealed worsening infection and interval development of a spinal epidural abscess. The patient underwent a CT-guided biopsy of the mass, which revealed $B$. henselae by PCR. The ultimate antibiotic regimen entailed a 12-week course of trimethoprim/ sulfamethoxazole. Tasher et al. reported a case of a 5-yearold boy diagnosed with a cervical epidural abscess, treated initially with cloxacillin, gentamicin, and ceftriaxone. ${ }^{11}$ However, due to worsening torticollis and evidence of spinal cord compression on follow-up imaging, the patient required surgical decompression. Intraoperative fluid PCR analysis revealed $B$. henselae. Antibiotic therapy initially included gentamicin and rifampin for 4 weeks, followed by azithromycin and rifampin for an additional 6 weeks.

The patient currently described reflects the same age demographic as prior reports of spinal epidural abscesses due to cat scratch disease. Similar to prior presentations, our patient initially received a diagnosis of cat scratch disease by serology and had been undergoing treatment when new symptoms prompted further evaluation with radiological imaging. The role of antibiotic therapy in the treatment of cat scratch disease is unclear. For regional lymphadenitis following inoculation of the skin at a distal site, the infection is usually self-limited. When treated with antibiotics, azithromycin is commonly used and is recommended by the Infectious Diseases Society of America based on the results of a single, small, randomized controlled trial..$^{3,10}$ In disseminated disease, including hepatosplenic granulomata, endocarditis, neuroretinitis, and osteomyelitis, the optimal antibiotic regimen is not known. Although there is no established treatment regimen for vertebral osteomyelitis with or without epidural abscess, we chose a combination regimen of doxycycline and rifampin. This decision was based on the limited published data of $B$. henselae vertebral osteomyelitis in adults, ${ }^{4,5}$ as well as studies and recommendations for treating other serious $B$. henselae infections, including endocarditis and CNS disease. ${ }^{2,8,9}$ The addition of rifampin in this case and in previous reports ${ }^{5,6,11}$ appears to enhance the treatment regimen in patients with vertebral osteomyelitis. Furthermore, early transition to oral antibiotics, within a week in this case, still provided for a successful outcome.

This report describes only the third case of biopsyproven $B$. henselae vertebral osteomyelitis with spinal epidural abscess. Surgical intervention has only been required in 2 of the 4 reported cases of epidural abscess due to cat scratch disease. While serology studies and immunoglobulin assays aid the diagnosis of cat scratch disease, direct sampling (via CT-guided biopsy or open surgery) provides for diagnosis confirmation and provides valuable information regarding antibiotic therapy. Prompt diagnosis and appropriate treatment of this rare cause of spinal epidural abscess are imperative to avoid progressive spinal cord compression and potentially devastating neurological sequelae.

\section{References}

1. Abdel-Haq N, Abuhammour W, Al-Tatari H, Asmar B: Disseminated cat scratch disease with vertebral osteomyelitis and epidural abscess. South Med J 98:1142-1145, 2005

2. Baltimore RS, Gewitz M, Baddour LM, Beerman LB, Jackson MA, Lockhart PB, et al: Infective endocarditis in childhood: 2015 update: a scientific statement from the American Heart Association. Circulation 132:1487-1515, 2015

3. Bass JW, Freitas BC, Freitas AD, Sisler CL, Chan DS, Vincent JM, et al: Prospective randomized double blind placebo-controlled evaluation of azithromycin for treatment of cat-scratch disease. Pediatr Infect Dis J 17:447-452, 1998

4. Graveleau J, Grossi O, Lefebvre M, Redon H, Caignon JM, Pallardy A, et al: Vertebral osteomyelitis: an unusual presentation of Bartonella henselae infection. Semin Arthritis Rheum 41:511-516, 2011

5. Grossi O, Denoyel GA, Redon H, Caignon JM, Généreau T, de Faucal P: Challenges in the diagnosis of culture negative 
vertebral osteomyelitis in adults: case of Bartonella henselae infection. Joint Bone Spine 80:671-673, 2013

6. Hawkins M, Bolton M: Pediatric spinal epidural abscess: a 9-year institutional review and review of the literature. Pediatrics 132:e1680-e1685, 2013

7. Hussain S, Rathore MH: Cat scratch disease with epidural extension while on antimicrobial treatment. Pediatr Neurosurg 43:164-166, 2007

8. Reed JB, Scales DK, Wong MT, Lattuada CP Jr, Dolan MJ, Schwab IR: Bartonella henselae neuroretinitis in cat scratch disease. Diagnosis, management, and sequelae. Ophthalmology 105:459-466, 1998

9. Rolain JM, Brouqui P, Koehler JE, Maguina C, Dolan MJ, Raoult D: Recommendations for treatment of human infections caused by Bartonella species. Antimicrob Agents Chemother 48:1921-1933, 2004

10. Stevens DL, Bisno AL, Chambers HF, Dellinger EP, Goldstein EJ, Gorbach SL, et al: Practice guidelines for the diagnosis and management of skin and soft tissue infections: 2014 update by the Infectious Diseases Society of America. Clin Infect Dis 59:e10-e52, 2014 (Erratum in Clin Infect Dis 60:1448, 2015)

11. Tasher D, Armarnik E, Mizrahi A, Liat BS, Constantini S, Grisaru-Soen G: Cat scratch disease with cervical vertebral osteomyelitis and spinal epidural abscess. Pediatr Infect Dis J 28:848-850, 2009

\section{Disclosures}

The authors report no conflict of interest concerning the materials or methods used in this study or the findings specified in this paper.

\section{Author Contributions}

Conception and design: all authors. Acquisition of data: Dornbos, Morin. Analysis and interpretation of data: Dornbos, Watson, Pindrik. Drafting the article: Dornbos, Morin. Critically revising the article: Dornbos, Watson, Pindrik. Reviewed submitted version of manuscript: all authors. Approved the final version of the manuscript on behalf of all authors: Dornbos. Study supervision: Pindrik.

\section{Correspondence}

David Dornbos III, Department of Neurological Surgery, The Ohio State University Wexner Medical Center, N1014 Doan Hall, 410 W 10th Ave., Columbus, OH 43210. email: david. dornbos@osumc.edu. 